Article

\title{
Power Decoupling Method Based on the Diagonal Compensating Matrix for VSG-Controlled Parallel Inverters in the Microgrid
}

\author{
Bin Li* (D) and Lin Zhou \\ State Key Laboratory of Power Transmission Equipment \& System Security and New Technology, \\ Chongqing University, Shapingba District, Chongqing 400044, China; zhoulin@cqu.edu.cn \\ * Correspondence: lbsas@163.com; Tel.: +86-185-1232-5075
}

Received: 19 November 2017; Accepted: 13 December 2017; Published: 17 December 2017

\begin{abstract}
The thought of the virtual synchronous generator (VSG) for controlling the grid-connected inverters and providing virtual inertia to the microgrid is emerging as a wide extension of the droop control, power coupling that always exists in the low-voltage microgrid, which may deteriorate the dynamic response and the stability of the system. In this paper, the principle of VSG control is introduced first. As an important issue of VSG control, the mechanism of the power coupling in the low-voltage microgrid is analyzed and the small-signal equivalent model of the power transmission loop is established. Subsequently, a power decoupling method based on the diagonal compensating matrix for VSG is proposed, which can realize the power decoupling with no impact on the original control channel. Meanwhile, the feasibility analysis of the decoupling method and the improved approach for reactive power sharing are also discussed. Simulation results verify the effectiveness of the decoupling strategy for VSGs.
\end{abstract}

Keywords: virtual synchronous generator (VSG); power decoupling; diagonal compensating matrix; small signal model

\section{Introduction}

A microgrid is an organic system composed of loads, distributed generations (DGs), and energy storage devices. Relative to the main network, a microgrid is an intelligent controlled unit. For customers, a microgrid can realize self-control, self-protection, and self-management in a flexible manner. It can meet the demands of a variety of load and specific power quality requirements $[1,2]$. In a microgrid, the inverter is one of the most important components. Yet it presents some drawbacks, such as the small output impedance, weak ability of overload, and lack of inertia [3,4]. The virtual synchronous generator (VSG) algorithm has been proposed to alleviate the above drawbacks by simulating the inertia element of the synchronous generator. As the extension of droop control, VSG enables it to respond to the abnormal frequency of the grid side and improves the stability of the power system [5-8].

Low-voltage microgrid lines present resistive or resistance-inductance properties, which can lead to strong coupling between active and reactive power outputs. This phenomenon may cause considerable impacts on the normal operation of microgrid, grid-connected control, and the power allocation among the DGs. Hence, it is important to investigate the power coupling effect for the improvement of operational stability and economic efficiency of the microgrid [9].

In engineering practice, large inductance has been introduced to reduce the coupling extent. However, this effort increases the line voltage drop and the total cost. Traditional VSG control has not paid much attention to power decoupling. Research on the independent control between active and reactive powers has mostly been based on droop control. The specific decoupling methods can be 
divided into three categories. The first one is based on the coordinate transformation. A coordinate rotation matrix can be established according to the impedance angle of the line, and is used to transform the real power flows [9-11] into decoupled virtual power flows. Similar results have also been developed to transform the output voltage into decoupled virtual voltage [12,13]. This approach can only realize the decoupling of the virtual power output, but not the real power output. Since the equivalent droop equations have been changed, the microgrid cannot be directly accessed by the distribution network that contains the traditional power generation, such as a steam turbine or diesel-driven generator. Moreover, complexity increases for the power control.

The second category is the virtual impedance-based decoupling control [14-18]. Through introducing a virtual inductance and subtracting its voltage drop at the input side of the voltage loop, the system impedance can be adjusted for power decoupling. Although this approach is simple, a large virtual inductance is required in the resistive environment, which can aggravate the line voltage drop and causes harmonic pollution.

The inversion of droop control is categorized as the third type $[19,20]$. Traditional droop control contains an active power channel $(P-f)$ and a reactive power channel $(Q-V)$, which exists strong coupling between the power outputs in the microgrid. Hence, some scholars have proposed the $P-V$ and $Q-f$ droop control. This creates a good power decoupling effect in the resistive environment. However, it is only appropriate for the case in which the line resistive value is far greater than its inductance value. Moreover, the microgrid cannot be directly accessed by the distribution network that contains the traditional power generation, such as a steam turbine or diesel-driven generator, because the equivalent droop equations have been changed.

All three approaches do not realize the decoupling control by acting on the coupling component directly. Thus, they can be considered as the indirect decoupling method. This paper proposes a power decoupling method, which directly compensates for the coupling component of the power loop. The decoupling is attained by use of a decoupling matrix in the VSG control unit and making the power transfer matrix a diagonal matrix. This method is based on the linearized power transfer model. It possesses a simple structure and better stability compared to the existing decoupling methods. In addition, the proposed power decoupling method is suitable for power decoupling in the microgrid, which has variable loads and frequent small power fluctuations.

The rest of this paper is organized as follows. The principle of the VSG control method is described in Section 2. The power/frequency and excitation controller are also introduced. In Section 3, the mechanism of power coupling is studied and the small signal model of the power loop is established. The proposed decoupling method based on the diagonal decoupling matrix for VSG is analyzed in Section 4 . The feasibility analysis and improvement for power sharing are given. Simulation results are presented in Section 5 to demonstrate the effectiveness of the proposed method, followed by the conclusion in Section 6.

\section{VSG Control Principle}

The main structure of one grid-connected inverter using the VSG control strategy is shown in Figure 1. A distributed generator (DG) with stored energy is located at the direct current (DC) side. For simplicity of analysis, a DC source is used here instead. The three-phase voltage-source inverter is adopted in the main circuit. $V_{\mathrm{dc}}$ is the voltage of the DC source; $Z_{\text {line }}$ is the line impedance from DG to the point of common coupling (PCC); $R_{\mathrm{in}}, L$, and $C$ denote the inductance internal resistance, the smoothing inductance, and the smoothing capacitance of the filter, respectively; $v_{\mathrm{o}}$ and $i_{\mathrm{o}}$ are the filter capacitor voltage and the inverter's output current, respectively; $i_{c}$ denotes the filter capacitor current; $P_{\text {set }}$ and $Q_{\text {set }}$ represent the input mechanical power and reactive power reference of the inverter, 
respectively; and $P_{\mathrm{e}}$ and $Q_{\mathrm{e}}$ represent the active and reactive power output of the inverter, respectively. In this article, according to the instantaneous power theory, $P_{\mathrm{e}}$ and $Q_{\mathrm{e}}$ can be calculated as:

$$
\begin{aligned}
& P_{\mathrm{e}}=v_{\mathrm{O} \_\alpha} i_{\mathrm{O} \_\alpha}+v_{\mathrm{O} \_\beta} i_{\mathrm{O} \_\beta} \\
& Q_{\mathrm{e}}=v_{\mathrm{O} \_\beta} i_{\mathrm{O}_{-} \alpha}-v_{\mathrm{O} \_} i_{\mathrm{O} \_} \beta
\end{aligned}
$$

The outer power loop generates the command voltage, which is used as the input signal of the voltage loop by controlling the instantaneous active and reactive power of the inverter. To ensure the accuracy, the voltage loop adopts the quasi-proportional-resonant (quasi-PR) controller which is characterized by an excellent tracking performance.

Figure 1 also shows block diagrams of the active power loop and the reactive power loop. The active power loop mainly simulates the rotor motion equation of synchronous generator (SG). A second-order model of SGs can be expressed as [8]:

$$
\left\{\begin{array}{l}
J \frac{\mathrm{d} \omega}{\mathrm{d} t}=\frac{P_{\mathrm{set}}}{\omega_{\mathrm{N}}}-\frac{P_{\mathrm{e}}}{\omega_{\mathrm{N}}}-D_{\mathrm{p}}\left(\omega-\omega_{\text {ref }}\right) \\
\frac{\mathrm{d} \delta}{\mathrm{d} t}=\omega
\end{array}\right.
$$

where $\delta$ is the power angle of the $\mathrm{SG}, \omega$ is the electric angular frequency of the $\mathrm{SG}, J$ is the moment of inertia of the $S G, D_{p}$ is the damping coefficient representing the function of damping winding, $\omega_{\text {ref }}$ is the synchronization angular frequency of the power grid, and $\omega_{\mathrm{N}}$ is the rated angular frequency. During normal operation, the angular frequency reference $\omega_{\text {ref }}$ is equal to $\omega_{N}$, which can implement the primary frequency modulation (PFM) of VSG. For the design of the active power loop of VSG, most existing researches on the design of the VSG active power loop are based on the PFM function.

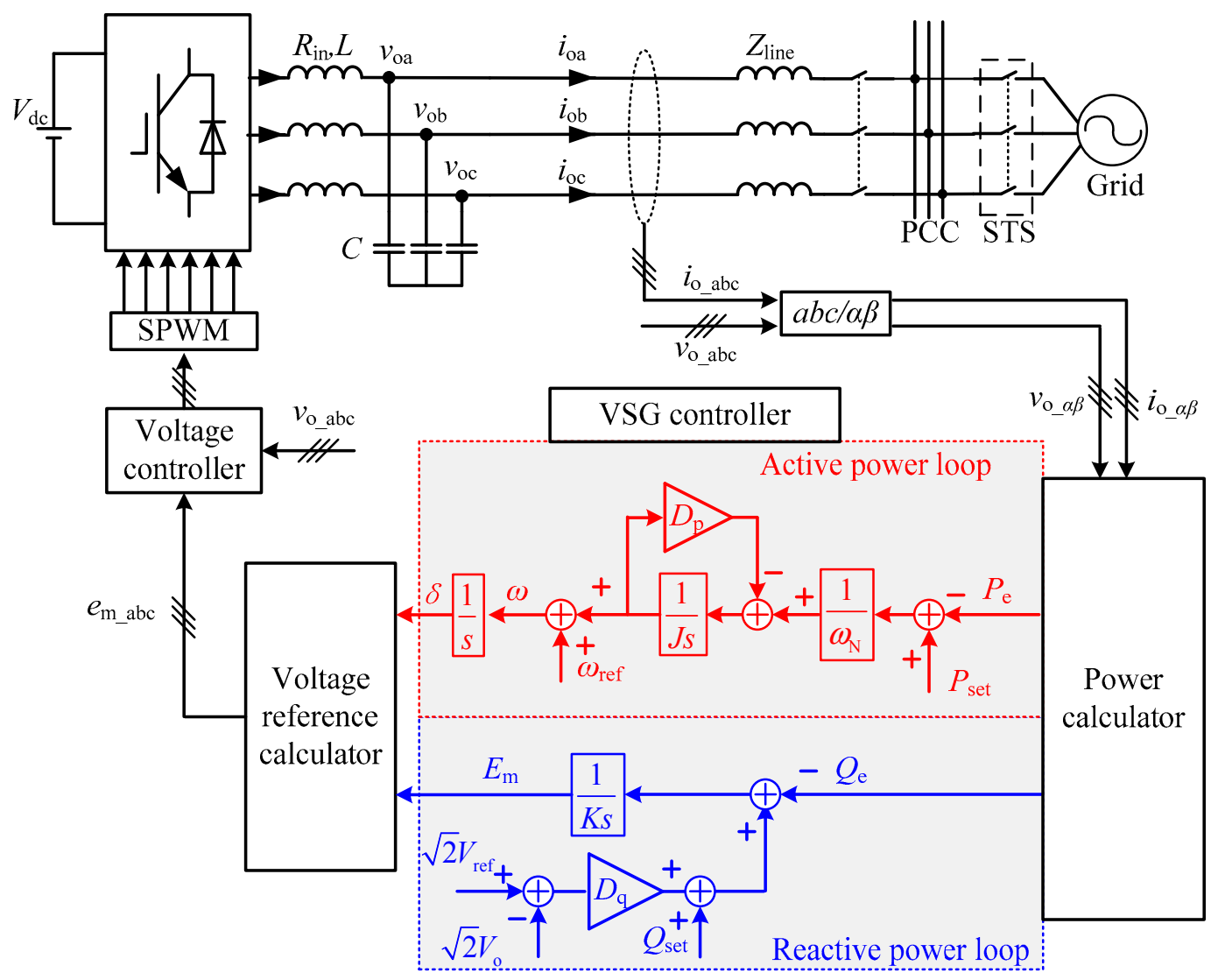

Figure 1. Control diagram of the inverter based on virtual synchronous generator (VSG). 
The reactive power loop of VSG contains the floating voltage and the reactive power controlled unit. Its control operation can be expressed as:

$$
K \frac{\mathrm{d} E_{\mathrm{m}}}{\mathrm{d} t}=Q_{\text {set }}+\sqrt{2} D_{\mathrm{q}}\left(V_{\text {ref }}-V_{\mathrm{o}}\right)-Q_{\mathrm{e}}
$$

where $D_{\mathrm{q}}$ is the droop coefficient of voltage, $K$ is the reactive power regulation coefficient, and $V_{\mathrm{o}}$ and $V_{\text {ref }}$ are effective values of the output voltage and rated voltage, respectively. The input command signal $e_{\mathrm{m}}$ of the voltage loop can be obtained by combining the amplitude information $E_{\mathrm{m}}$ generated by the reactive power loop and the phase information $\delta$ by the active power loop as:

$$
\left\{\begin{array}{l}
e_{\mathrm{m} \_\mathrm{a}}=\sqrt{2} E_{\mathrm{m}} \sin \delta \\
e_{\mathrm{m} \_\mathrm{b}}=\sqrt{2} E_{\mathrm{m}} \sin \left(\delta-\frac{2}{3} \pi\right) \\
e_{\mathrm{m} \_\mathrm{c}}=\sqrt{2} E_{\mathrm{m}} \sin \left(\delta+\frac{2}{3} \pi\right)
\end{array}\right.
$$

\section{Output Power Model of Microgrid}

To facilitate the analysis and ensure the generality, the simplified equivalent circuit of a micro-source is illustrated in Figure 2.

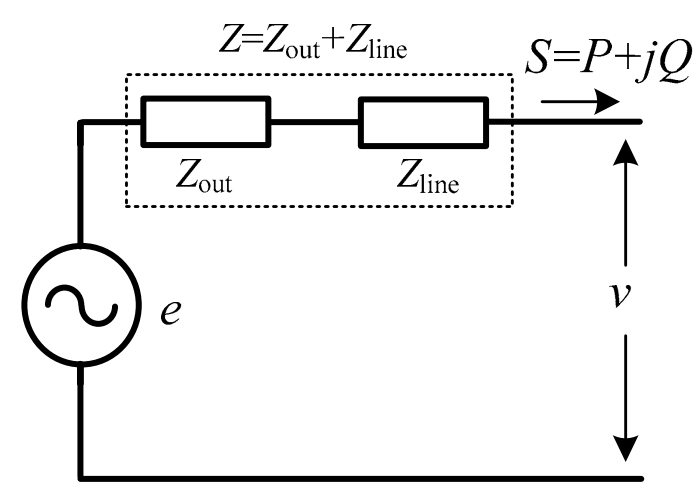

Figure 2. Equivalent circuit of an inverter under the grid-connected mode.

In Figure 2, the micro-source is equivalent to a controlled voltage source. $S=P+j Q$ is the line power of the microgrid. $e$ is the output voltage of the micro-source and $v$ is the voltage of PCC. The phasor form can be written as $E \angle \delta$ and $V \angle 0$. The grid impedance is represented by $Z$, which consists of the output impedance $Z_{\text {out }}$ and the line impedance $Z_{\text {line }}$. Suppose that the grid impedance is referred to as $Z \angle \theta=R+j X$. A single phase study is given below. The output power equation can be written as:

$$
\left\{\begin{array}{l}
P=\frac{V}{Z}[E \cos (\theta-\delta)-V \cos \theta] \\
Q=\frac{V}{Z}[E \sin (\theta-\delta)-V \sin \theta]
\end{array}\right.
$$

In a high-voltage or mid-voltage power grid, the line impedance is generally considered to be inductive $\left(\theta=90^{\circ}\right)$ because the impedance due to line inductance is much larger than that due to line resistance. Moreover, the phase angle difference $\delta$ is normally under $30^{\circ}$ for a synchronous generator and in this case, $\sin \delta \approx \delta$ and $\cos \delta \approx 1$. Then, (5) can be simplified as:

$$
\left\{\begin{array}{l}
P=\frac{E V}{Z} \delta \\
Q=\frac{V(E-V)}{Z}
\end{array}\right.
$$

Consequently, the active power can be regulated by adjusting the phase angle $\delta$ and the reactive power can be regulated by adjusting the amplitude of the inverter output voltage, which is the basis of the droop characteristics of VSG. However, in the low-voltage microgrid, there is large resistance 
in the connecting line. The approximate decoupling cannot be obtained and strong coupling exists between active and reactive power controls. In this paper, to solve this problem, the linearized model of output power is established first.

The output power deviation matrix caused by the respective deviations $\hat{E}$ and $\hat{\delta}$ at the quiescent point $\left(E_{\mathrm{s}}, \delta_{\mathrm{s}}\right)$ can be expressed as:

$$
\left[\begin{array}{c}
\hat{P} \\
\hat{Q}
\end{array}\right]=\frac{V}{Z}\left[\begin{array}{cc}
E_{\mathrm{s}} \sin \left(\theta-\delta_{\mathrm{s}}\right) & \cos \left(\theta-\delta_{\mathrm{s}}\right) \\
-E_{\mathrm{s}} \cos \left(\theta-\delta_{\mathrm{s}}\right) & \sin \left(\theta-\delta_{\mathrm{s}}\right)
\end{array}\right]\left[\begin{array}{l}
\hat{\delta} \\
\hat{E}
\end{array}\right]
$$

Equation (7) represents the output power model and the associated $2 \times 2$ matrix is called the power transfer matrix (PTM). Then, the small-signal VSG control structure of the power loop can be derived from (2), (3), and (7) and is illustrated in Figure 3. The power coupling components in the dashed boxes can be observed clearly. When the impedance angle $\theta$ decrease, the coupling components between the active and reactive power will increase. Moreover, the coupling component between the active power and the voltage amplitude is much smaller than that between the reactive power and the angular frequency.

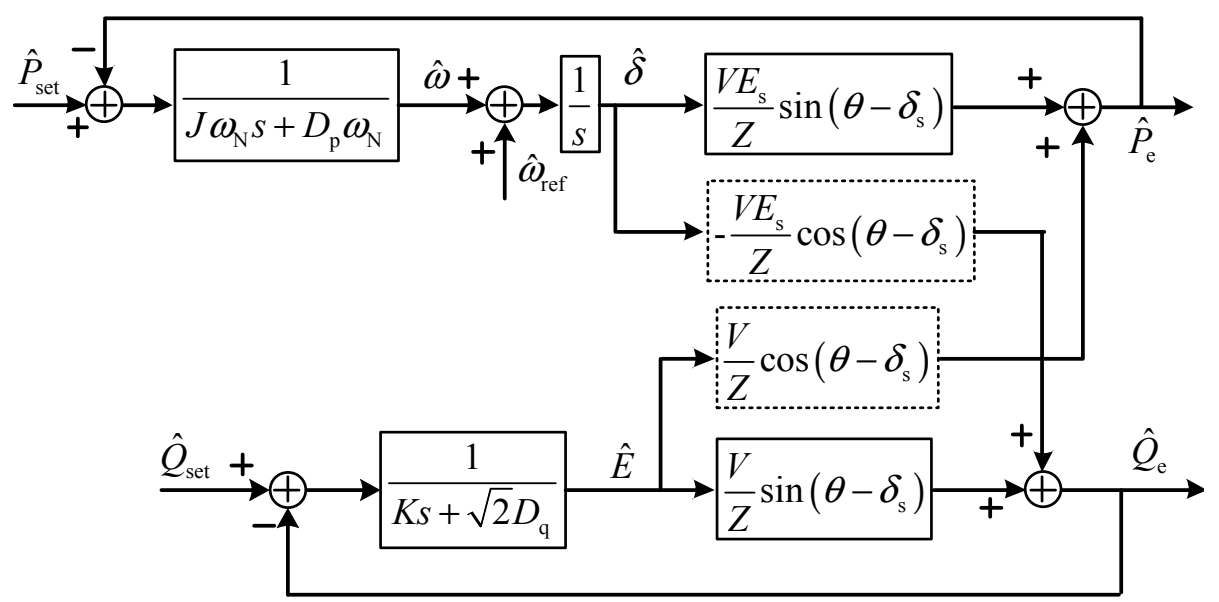

Figure 3. Small-signal equivalent model of VSG.

\section{Decoupling Control Method for VSG Control}

This paper intends to compensate for the coupling component by adopting a direct power decoupling method. Direct decoupling control methods have two popular types: dynamic decoupling and static decoupling. Dynamic decoupling requires that the channels are decoupled in the transition process and in the steady-state process. This method deeply relies on the mathematical model of the system and cannot attain the desired effect if the model experiences errors or parameter perturbation. Static decoupling only requires the channels to be decoupled in the steady-state process. Although it may degrade the decoupling performance and incurs dynamic deviation, it has a lower sensitivity to model error and parameter perturbation. Thus, this paper adopts the static decoupling method.

\subsection{Control Principle}

A feed-forward decoupling compensator for a two-input and two-output (TITO) system was introduced to obtain the approximate decoupling [21]. However, the influences on the original coupling channel in series with the compensator are not considered. This may change the proportional gain of the original channel and affect the stability of the power control loop. In the previous section, the power independent control can be realized when the PTM is a diagonal matrix. Borrowing from this thought and the modern control theory, a diagonal decoupling matrix is introduced. 
It follows from (6) and Figure 3 that the coupling channels of the output power can be regarded as a TITO system. Inputs are $\hat{E}$ and $\hat{\delta}$ and outputs are $\hat{P}_{\mathrm{e}}$ and $\hat{Q}_{\mathrm{e}}$ in the complex frequency domain. Static decoupling requires that the transfer function matrix be a nonsingular diagonal constant matrix. The PTM of Equation (7) can be expressed as:

$$
G_{\mathrm{s}}=\frac{V}{Z}\left[\begin{array}{cc}
E_{\mathrm{s}} \sin \left(\theta-\delta_{\mathrm{s}}\right) & \cos \left(\theta-\delta_{\mathrm{s}}\right) \\
-E_{\mathrm{s}} \cos \left(\theta-\delta_{\mathrm{s}}\right) & \sin \left(\theta-\delta_{\mathrm{s}}\right)
\end{array}\right]
$$

The decoupled transfer matrix can be expressed as a diagonal matrix

$$
W=\frac{V}{Z}\left[\begin{array}{cc}
E_{\mathrm{s}} \sin \left(\theta-\delta_{\mathrm{s}}\right) & 0 \\
0 & \sin \left(\theta-\delta_{\mathrm{s}}\right)
\end{array}\right]
$$

Then, the transfer function of controlled object in series with the decoupling link $G_{\mathrm{c}}$ becomes

$$
\left[\begin{array}{c}
\hat{P} \\
\hat{Q}
\end{array}\right]=G_{\mathrm{s}} G_{\mathrm{c}}\left[\begin{array}{l}
\hat{\delta} \\
\hat{E}
\end{array}\right]=W\left[\begin{array}{l}
\hat{\delta} \\
\hat{E}
\end{array}\right]
$$

$G_{\mathrm{C}}$ can be calculated as:

$$
\begin{gathered}
G_{\mathrm{c}}=G_{\mathrm{s}}^{-1} W=\left[\begin{array}{cc}
\frac{V E_{\mathrm{s}}}{Z} \sin \left(\theta-\delta_{\mathrm{s}}\right) & \frac{V}{Z} \cos \left(\theta-\delta_{\mathrm{s}}\right) \\
-\frac{V E_{\mathrm{s}}}{Z} \cos \left(\theta-\delta_{\mathrm{s}}\right) & \frac{V}{Z} \sin \left(\theta-\delta_{\mathrm{s}}\right)
\end{array}\right]_{-\sin \left(\theta-\delta_{\mathrm{s}} \cos \left(\theta-\delta_{\mathrm{s}}\right)\right.}^{-1}\left[\begin{array}{cc}
\frac{V E_{\mathrm{s}}}{Z} \sin \left(\theta-\delta_{\mathrm{s}}\right) & 0 \\
E_{\mathrm{s}} & \frac{V}{\mathrm{Z}} \sin \left(\theta-\delta_{\mathrm{s}}\right)
\end{array}\right] \\
=\left[\begin{array}{cc}
\sin ^{2}\left(\theta-\delta_{\mathrm{s}}\right) & \frac{\sin ^{2}\left(\theta-\delta_{\mathrm{s}}\right)}{E_{\mathrm{s}} \sin \left(\theta-\delta_{\mathrm{s}}\right) \cos \left(\theta-\delta_{\mathrm{s}}\right)}
\end{array}\right] \\
=\left[\begin{array}{ll}
G_{c 11} & G_{c 12} \\
G_{c 21} & G_{c 22}
\end{array}\right]
\end{gathered}
$$

It is observed from (11) that only the impedance angle is needed and the definite length of the distributed line is not required. This method is based on compensating for the coupling component. Figure 4 shows the control diagram of the power loop in series with the decoupling link.

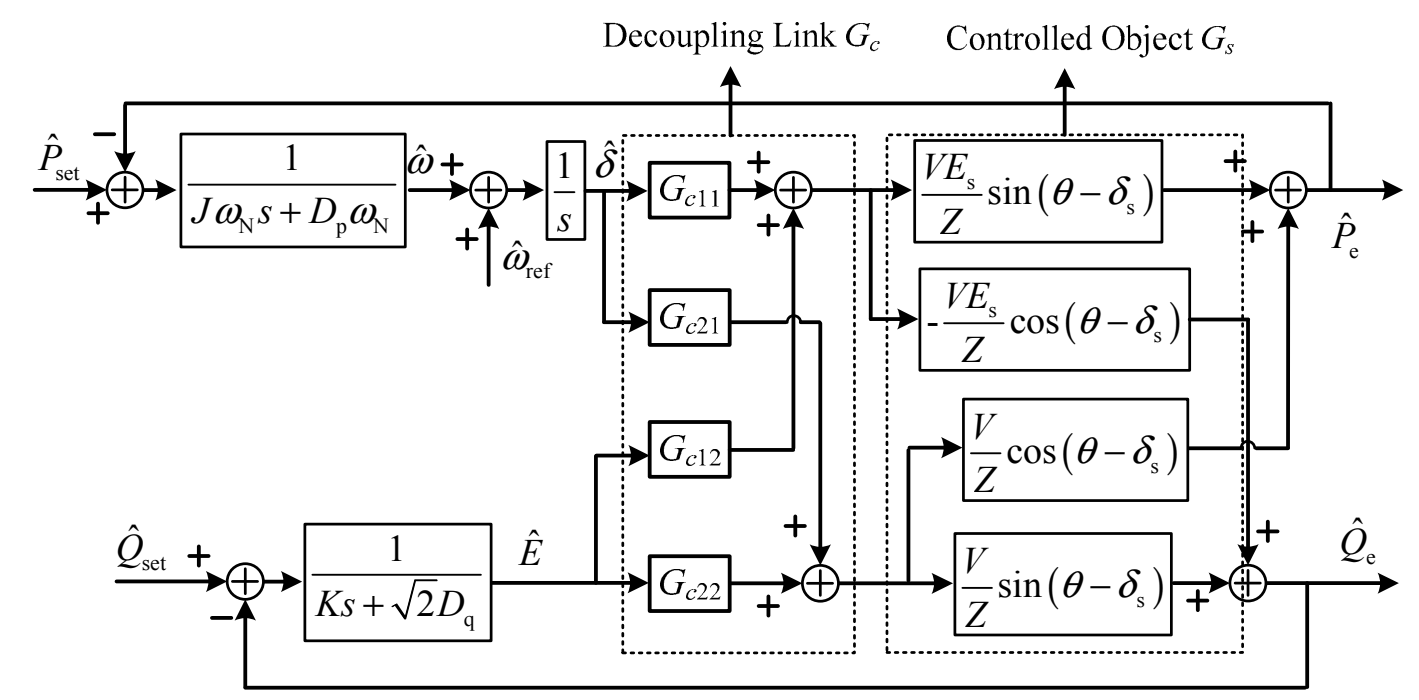

Figure 4. Control diagram of the power loop in series with the decoupling link. 


\subsection{Feasibility Analysis and Improvement Approach for Power Sharing}

A diagonal compensated matrix has been introduced to attain power decoupling based on the quiescent operating point. According to the relative gain array (RGA) method of the decoupling control [21], the RGA of the $G_{\mathrm{c}}$ can be derived as:

$$
\Lambda=\left[\begin{array}{cc}
\lambda_{11}=\frac{G_{c 11} G_{c 22}}{G_{c 11} G_{c 22}-G_{c 12} G_{c 21}} & \lambda_{12}=1-\lambda_{11} \\
\lambda_{21}=1-\lambda_{11} & \lambda_{22}=\lambda_{11}
\end{array}\right]=\left[\begin{array}{cc}
\sin ^{2}\left(\theta-\delta_{\mathrm{s}}\right) & \cos ^{2}\left(\theta-\delta_{\mathrm{s}}\right) \\
\cos ^{2}\left(\theta-\delta_{\mathrm{s}}\right) & \sin ^{2}\left(\theta-\delta_{\mathrm{s}}\right)
\end{array}\right]
$$

From (12), the coefficients of RGA always vary in $(0,1)$, which means that the introduction of the decoupling link cannot affect the stability of the system. On the other hand, since (7) denotes the average values of the instantaneous power flows over half the fundamental frequency cycle, discussions can only reflect the characteristics of the system in the frequency range that is lower than two times the power frequency. The decoupling method based on the diagonal matrix is only applicable to the power regulation under small disturbance near the quiescent operating point. If the output voltage amplitude and power angle change over a wide range, the decoupling effect of the system may be reduced and it is necessary to dynamically adjust the coefficient of power decoupling link. Otherwise, the decoupling may be a failure. Nonetheless, due to its simple structure and better stability, this method is suitable for power decoupling in a microgrid which has a diversity of loads and frequent small power fluctuations.

The other issue considered by power decoupling control is power sharing. To guarantee the sharing of actual power, improved virtual power-based control with a unified rotation angle is proposed [9]. Because the transformation matrix is nonsingular, the actual power will be shared equally as long as the virtual power is evenly shared among parallel inverters. Although the limitation of the actual power sharing performance heavily relies on the consistence of line impedance angles of the inverters is overcome, when the transmission lines among parallel inverters are different, the power decoupling effect will still be diminished.

Introduction of the decoupling link does not change the gain of the original channel. When the system reaches the steady state, a simplified equation can be derived:

$$
P_{\text {set }}-P_{\mathrm{e}}=D_{\mathrm{p}} \omega_{\mathrm{N}}\left(\omega-\omega_{\mathrm{ref}}\right)
$$

Equation (13) describes the droop characteristic between the active power and frequency of VSG. The active power sharing can be achieved directly by making $D_{p}$ of each inverter proportional to its capacity. For $Q-V$ control, however, the output voltage of the inverter will be not same due to the existence of line voltage drop. This phenomenon affects the reactive power sharing. To overcome this problem, the PCC voltage is introduced as a consistent variable. Figure 5 shows the improved reactive power loop. The reactive power sharing can be ensured by making $D_{\mathrm{q}}$ of each inverter proportional to its capacity.

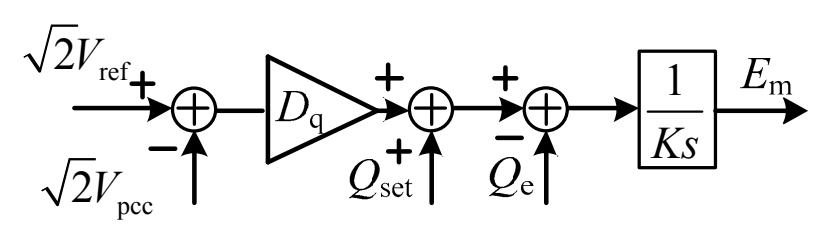

Figure 5. Improved $Q-V$ control loop. 


\section{Simulation Verification}

\subsection{Verification of the Power Decoupling Performance}

The proposed power decoupling method is verified by using MATLAB/Simulink in various respects. The main circuit and control scheme of one inverter are illustrated in Figure 1. Control parameters for the VSG controlled inverter are listed in Table 1 . The line impedance is $0.8+j 0.5 \Omega$.

Table 1. Electrical and control parameters.

\begin{tabular}{cccc}
\hline Parameters & Values & Parameters & Values \\
\hline$V_{\mathrm{dc}}$ & $800 \mathrm{~V}$ & $V_{\mathrm{pcc}}$ & $220 \mathrm{~V}$ \\
$L$ & $2 \mathrm{mH}$ & $P_{\text {set }}$ & $10 \mathrm{~kW}$ \\
$C$ & $300 \mu \mathrm{F}$ & $Q_{\text {set }}$ & $5 \mathrm{kVar}$ \\
$R_{\mathrm{in}}$ & $0.05 \Omega$ & $J$ & 0.2 \\
$\omega_{\mathrm{N}}$ & $314 \mathrm{rad} / \mathrm{s}$ & $D_{\mathrm{p}}$ & 20 \\
$E_{\mathrm{s}}$ & $235.7 \mathrm{~V}$ & $D_{\mathrm{q}}$ & 500 \\
$\delta_{\mathrm{s}}$ & 0.07 & $K$ & 50 \\
\hline
\end{tabular}

Two cases are considered. In Case 1, the frequency of the grid voltage is $50 \mathrm{~Hz}$ from $0 \mathrm{~s}<t<1 \mathrm{~s}$ and is changed to $49.9 \mathrm{~Hz}$ at $1.0 \mathrm{~s}$. Figure 6a shows the frequency waveform of the output voltage. In Case 2, a $0.02 \mathrm{pu}$ increase in the voltage amplitude of the distribution network occurs at $1 \mathrm{~s}$, as shown in Figure 6b.

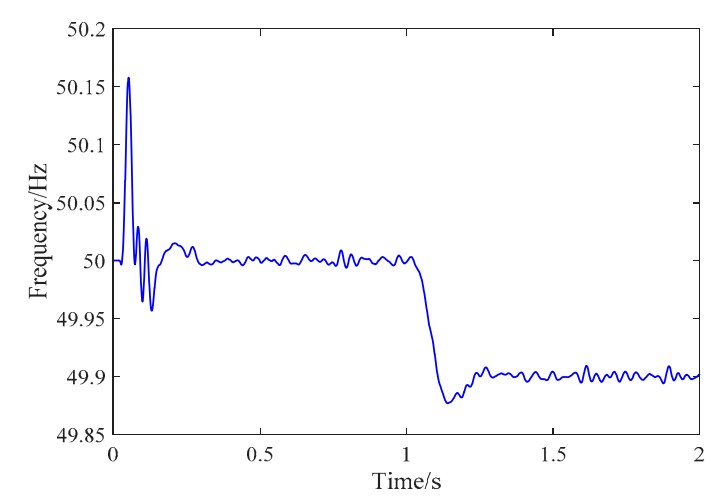

(a)

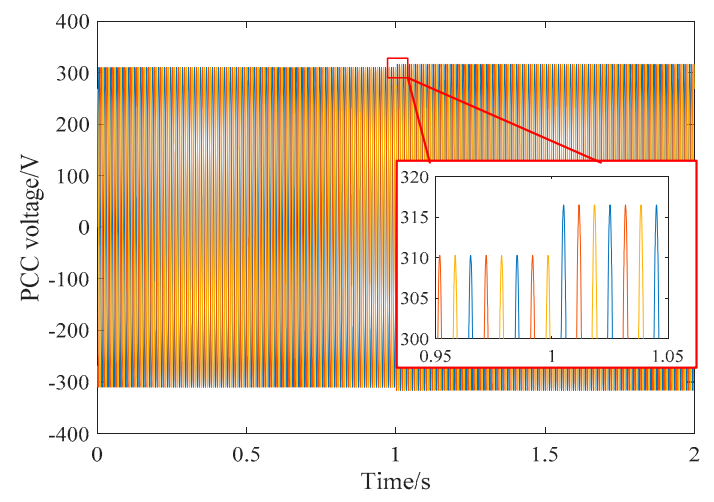

(b)

Figure 6. (a) The frequency waveform of the output voltage; (b) The PCC voltage waveform.

Figure 7a,b show the waveforms of line power without and with the power decoupling link, respectively. Plots in the left column correspond to the frequency deviation and that in the right column to the voltage deviation. Note that the line power is the power injected into the distribution network. The measured values are slightly smaller than the set values. After the frequency decreases, it can be seen from Figure $7 \mathrm{a}$ that the active power output increases by about $3.8 \mathrm{~kW}$, which means that VSG can change its active power output to respond to the frequency exception event of the grid-side. To benefit from the introduction of the swing Equation (2), the frequency of VSG control changes slowly, which means that VSG provides virtual inertia to the system to allow the frequency of VSG control to change slowly. This is beneficial to the stable operation of the power grid. Meanwhile, the impact of the frequency fluctuation on the reactive power decreases with the help of the power decoupling link. 

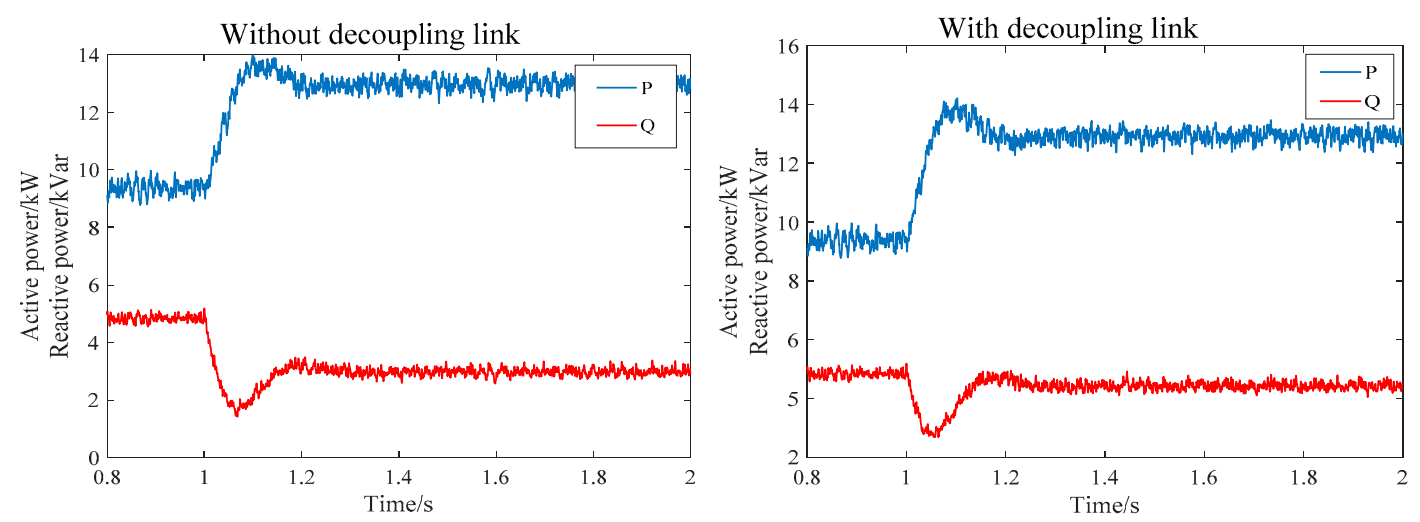

(a)
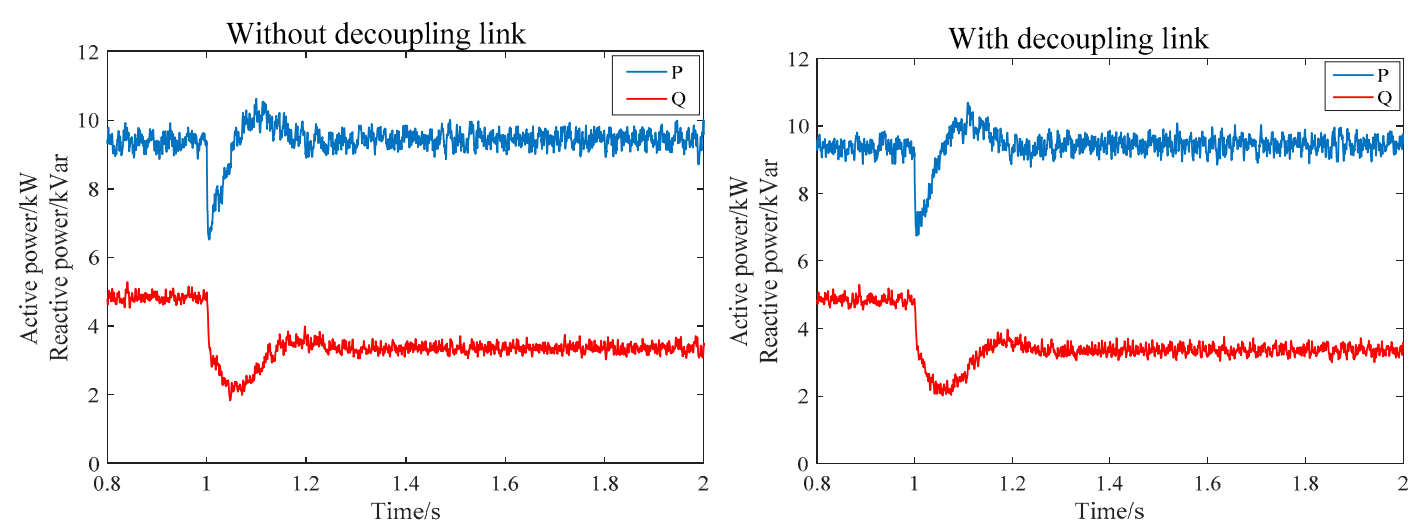

(b)

Figure 7. (a) The power output response to the frequency fluctuation; (b) The power output response to the voltage amplitude fluctuation.

It is observed from Figure $7 \mathrm{~b}$ that active and reactive power responses with and without power decoupling are almost the same. This indicates that the voltage fluctuation does not affect the active power and reactive power much. In other words, the coupling extent between the active power and voltage is less than that between the reactive power and frequency, which is consistent with the theoretical analysis in Section 3. It is observed from Figure 7b that VSG also responds to the voltage fluctuation.

\subsection{Verification of the Power Sharing Performance}

Figure 8 depicts the microgrid platform used in the simulation, which contains a $20 \mathrm{kVA}$ VSG and a $10 \mathrm{kVA}$ VSG. The microgrid is running under the grid-connected mode. The parameter values of respective VSGs are listed in Table 2. The line impedances of two DGs are different: one is $Z_{\text {Line1 }}=0.8+j 0.5 \Omega$, and another is $Z_{\text {Line2 }}=0.5+j 0.83 \Omega$.

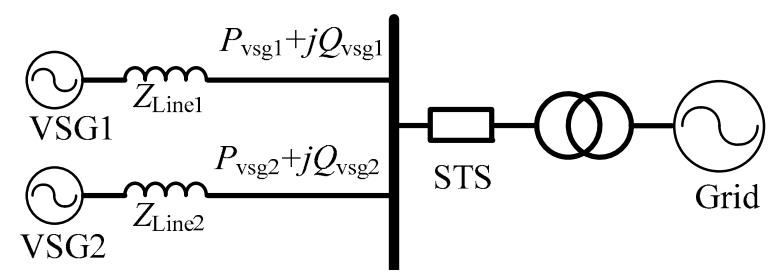

Figure 8. Microgrid simulation platform. 
Table 2. Electrical and control parameters of two virtual synchronous generators (VSGs).

\begin{tabular}{cccc}
\hline Parameters (VSG1) & Values & Parameters (VSG2) & Values \\
\hline$V_{\mathrm{dc}}$ & $800 \mathrm{~V}$ & $V_{\mathrm{dc}}$ & $600 \mathrm{~V}$ \\
$L$ & $3 \mathrm{mH}$ & $L$ & $2 \mathrm{mH}$ \\
$C$ & $400 \mu \mathrm{F}$ & $C$ & $300 \mu \mathrm{F}$ \\
$R_{\mathrm{in}}$ & $0.08 \Omega$ & $R_{\mathrm{in}}$ & $0.05 \Omega$ \\
$\omega_{\mathrm{N}}$ & $314 \mathrm{rad} / \mathrm{s}$ & $\omega_{\mathrm{N}}$ & $314 \mathrm{rad} / \mathrm{s}$ \\
$E_{\mathrm{s}}$ & $235.7 \mathrm{~V}$ & $E_{\mathrm{s}}$ & $230.1 \mathrm{~V}$ \\
$\delta_{\mathrm{s}}$ & 0.07 & $\delta_{\mathrm{s}}$ & 0.05 \\
$V_{\mathrm{pcc}}$ & $220 \mathrm{~V}$ & $V_{\mathrm{pcc}}$ & $220 \mathrm{~V}$ \\
$P_{\mathrm{set}}$ & $10 \mathrm{~kW}$ & $P_{\mathrm{set}}$ & $5 \mathrm{~kW}$ \\
$Q_{\mathrm{set}}$ & $5 \mathrm{kVar}$ & $Q_{\mathrm{set}}$ & $5 \mathrm{kVar}$ \\
$J$ & 0.15 & $J$ & 0.2 \\
$D_{\mathrm{p}}$ & 30 & $D_{\mathrm{p}}$ & 15 \\
$D_{\mathrm{q}}$ & 600 & $D_{\mathrm{q}}$ & 300 \\
$K$ & 55 & $K$ & 50 \\
\hline
\end{tabular}

Case 1: The frequency of grid voltage decreases by $0.1 \mathrm{~Hz}$ at $1.0 \mathrm{~s}$ and restores at $2.0 \mathrm{~s}$. Figure 9 shows the line powers of VSGs without and with the decoupling link. In Figure 9a, the two VSGs respond well to the frequency fluctuation and increase their active powers output according to their capacity ratios. This result verifies the active power sharing effect proposed in Section 4. It is also observed that the change of the active power results in the change of the reactive power. On the other hand, Figure $9 \mathrm{~b}$ illustrates that the decoupling link reduces the influence of the active power fluctuation on the reactive power. Figure 9c shows the output current waveform of VSG1 and the total harmonic distortion (THD) values verify the accuracy of the parameters selection.
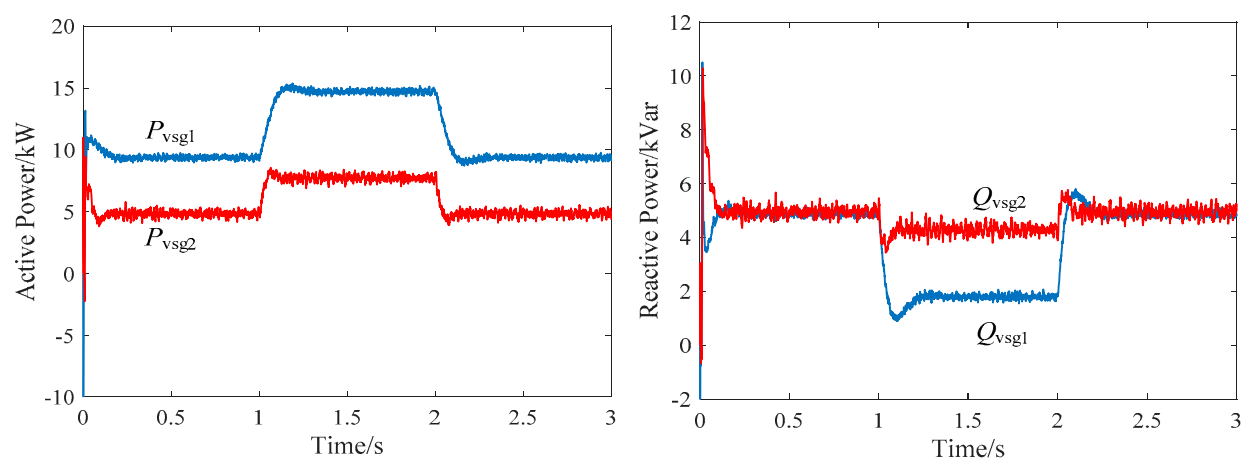

(a)
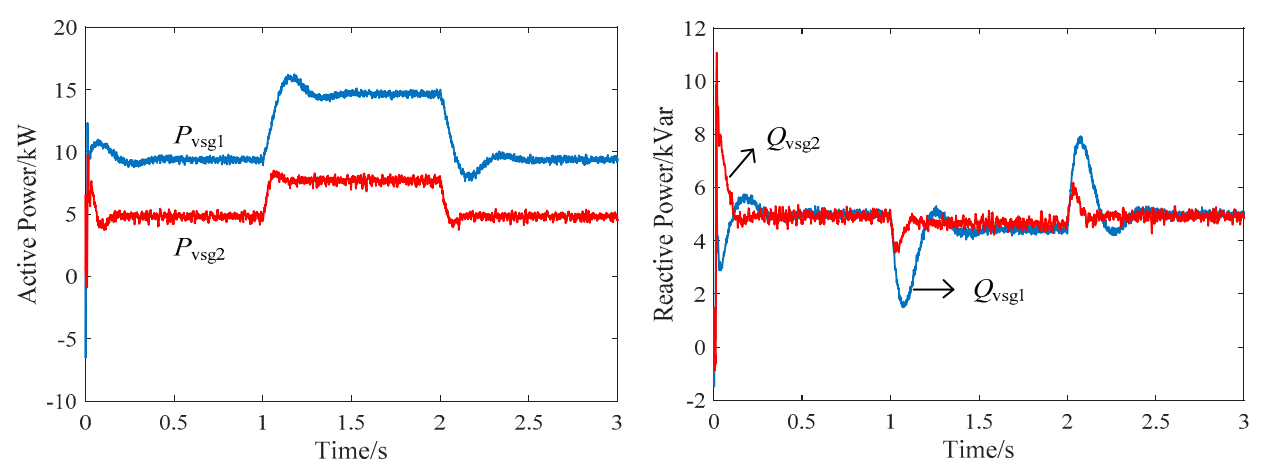

(b)

Figure 9. Cont. 


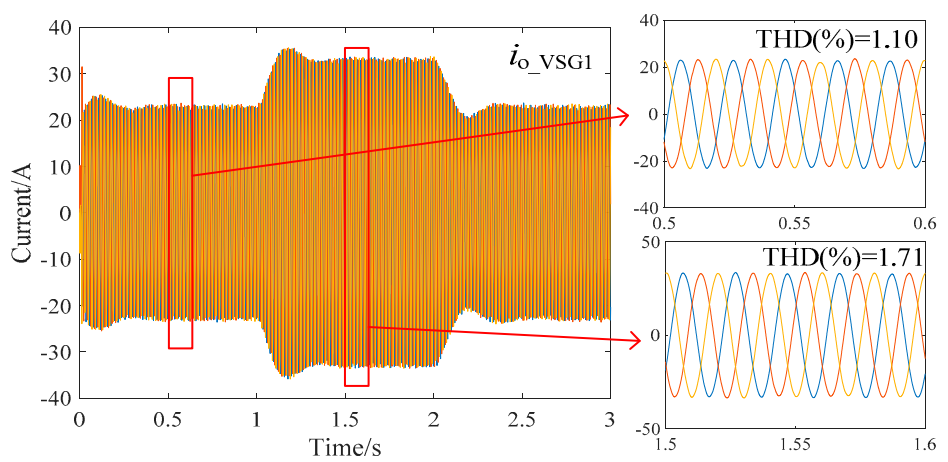

(c)

Figure 9. Output waveforms of VSG control to frequency deviation. (a) Without decoupling link; (b) With decoupling link; (c) Output current of VSG1 with decoupling link.

Case 2: The amplitude of the grid voltage decreases by $0.02 \mathrm{pu}$ at $1 \mathrm{~s}$ and restores at 2s. Figure 10 shows the line powers of VSGs without and with decoupling link. In Figure 10a, the two VSGs respond to the voltage fluctuation by adjusting their reactive power output. However, without the introduction of the PCC voltage, the two VSGs does not increase their reactive power output according to their capacity ratios. This situation has been improved in Figure 10b. Moreover, the change of the reactive power will not affect the active power output, which is consistent with the theoretical analysis in Section 3. Similarly, Figure 10c shows the output current waveform of VSG2 and the THD values verify the accuracy of the parameters selection.
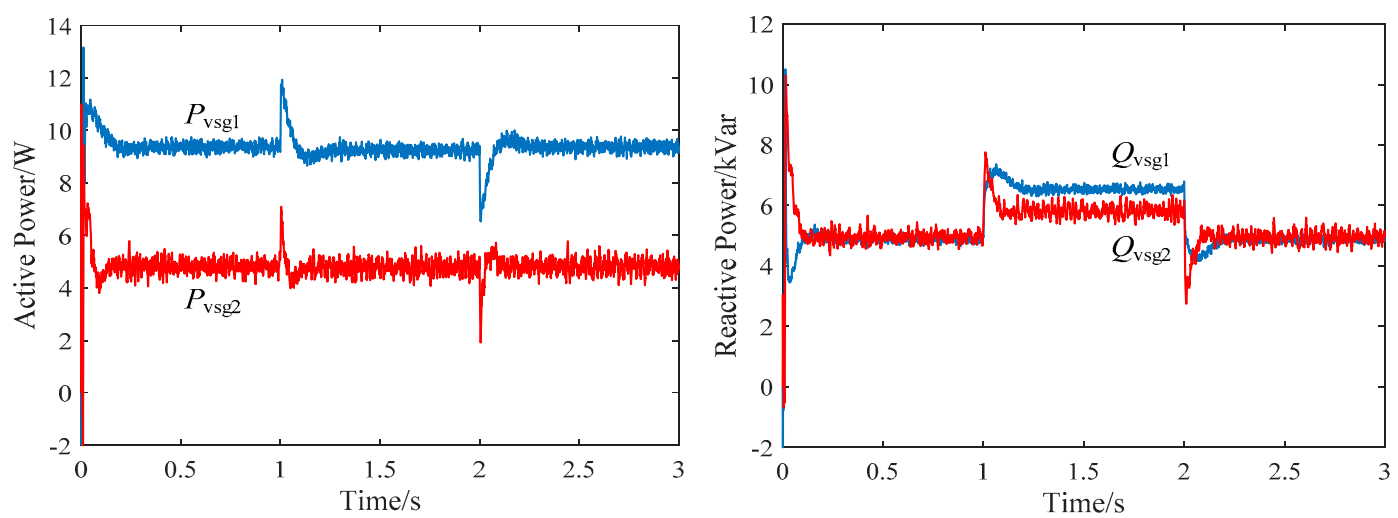

(a)
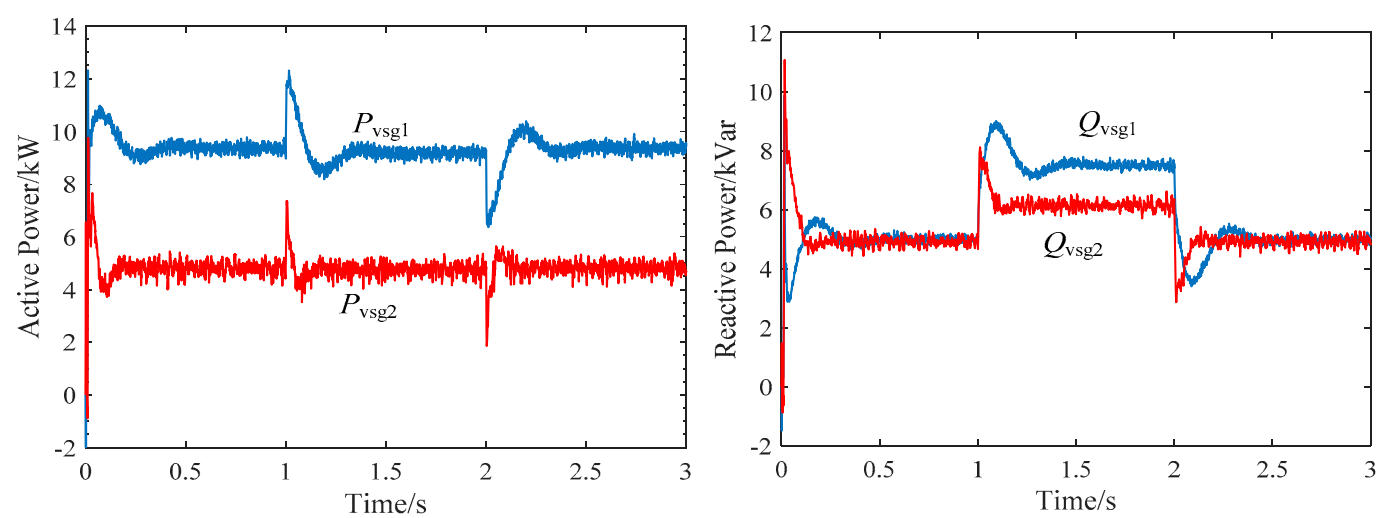

(b)

Figure 10. Cont. 


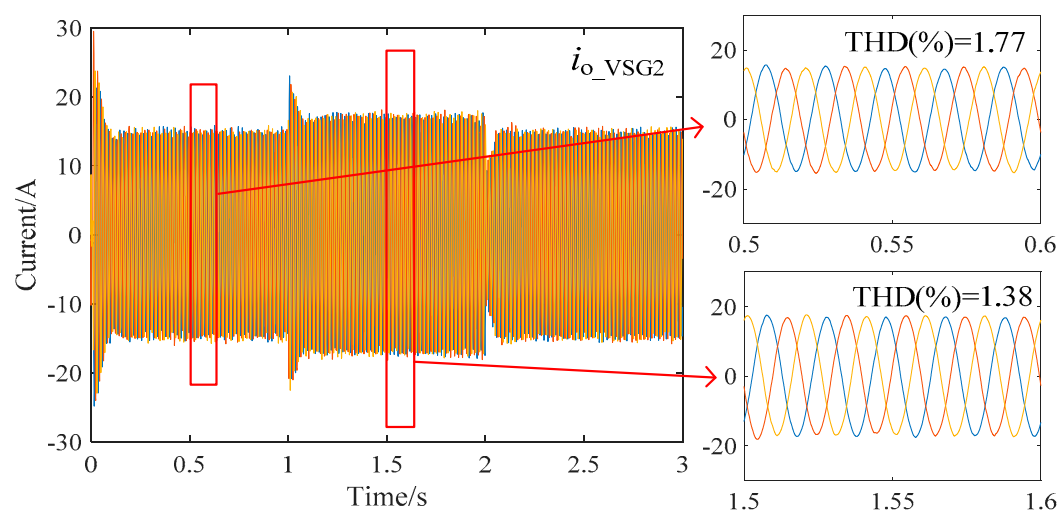

(c)

Figure 10. Output waveforms of VSG control to voltage deviation. (a) Without decoupling link; (b) With decoupling link; (c) Output current of VSG2 with decoupling link.

\section{Conclusions}

This paper has presented a power decoupling method based on the diagonal compensating matrix for VSG. By making the power transfer matrix a diagonal matrix, the nearly decoupling effect can be obtained. Compared with the traditional decoupling method, this new scheme requires no complex design and specific line parameter, which is helpful to the service for practical engineering. Moreover, power sharing among parallel inverters can be implemented with the introduction of the PCC voltage. Simulation results have demonstrated the merits of the proposed power decoupling control and the ability of VSG in the response to the grid abnormal events.

Acknowledgments: This work was supported by the National Natural Science Foundation of China (grant \#: 51477021) and the "111" State innovation \& Talent Recruitment Base (B08036).

Author Contributions: All authors contributed substantially to all aspects of this article.

Conflicts of Interest: The authors declare no conflict of interest.

\section{References}

1. Banerji, A.; Sen, D.; Bera, A.K. Microgrid: A review. In Proceedings of the Global Humanitarian Technology Conference: South Asia Satellite (GHTC-SAS), Trivandrum, India, 23-24 August 2013.

2. Lasseter, R.H. MicroGrids. In Proceedings of the IEEE Power Engineering Society Winter Meeting, New York, NY, USA, 27-31 January 2002.

3. Azmy, A.M.; Erlich, I. Impact of Distributed Generation on the Stability of Electrical Power Systems. In Proceedings of the Power Engineering Society General Meeting, San Francisco, CA, USA, 16 June 2005.

4. Aktarujjaman, M.; Haque, M.E.; Muttaqi, K.M.; Negnevitsky, M.; Ledwich, G. Control Stabilisation of Multiple Distributed Generation. In Proceedings of the Power Engineering Conference (AUPEC 2007), Perth, Australia, 9-12 December 2007.

5. Li, D.; Zhu, Q.; Lin, S. A Self-Adaptive Inertia and Damping Combination Control of VSG to Support Frequency Stability. IEEE Trans. Energy Convers. 2017, 32, 397-398. [CrossRef]

6. Suul, J.A.; Darco, S.; Guidi, G. Virtual Synchronous Machine-Based Control of a Single-Phase Bi-Directional Battery Charger for Providing Vehicle-to-Grid Services. IEEE Trans. Ind. Appl. 2016, 52, 3234-3244. [CrossRef]

7. Cvetkovic, I.; Boroyevich, D.; Burgos, R. Experimental Verification of a Virtual Synchronous Generator Control Concept. In Proceedings of the Control and Modeling for Power Electronics (COMPEL), Trondheim, Norway, 27-30 June 2016.

8. Wu, H.; Ruan, X.; Yang, D.; Chen, X.; Zhao, W.; Lv, Z.; Zhong, Q.C. Small-Signal Modeling and Parameters Design for Virtual Synchronous Generators. IEEE Trans. Ind. Electron. 2016, 63, 4292-4303. [CrossRef]

9. Wu, T.; Liu, Z.; Liu, J.; Wang, S. A Unified Virtual Power Decoupling Method for Droop-Controlled Parallel Inverters in Microgrids. IEEE Trans. Power Electron. 2016, 31, 5587-5603. [CrossRef] 
10. Rowe, C.N.; Summers, T.J.; Betz, R.E.; Cornforth, D.J. Arctan Power-Frequency Droop for Improved Microgrid Stability. IEEE Trans. Power Electron. 2013, 28, 3747-3759. [CrossRef]

11. De Brabandere, K.; Bolsens, B.; van den Keybus, J.; Woyte, A.; Driesen, J. A Voltage and Frequency Droop Control Method for Parallel Inverters. IEEE Trans. Power Electron. 2007, 22, 1107-1115. [CrossRef]

12. Li, Y.; Li, Y.W. Power Management of Inverter Interfaced Autonomous Microgrid Based on Virtual Frequency-Voltage Frame. IEEE Trans. Smart Grid 2011, 2, 30-40. [CrossRef]

13. Li, Y.; Li, Y.W. Virtual frequency-voltage frame control of inverter based low voltage microgrid. In Proceedings of the Electrical Power \& Energy Conference (EPEC), Montreal, QC, Canada, 22-23 October 2009.

14. Zhang, P.; Zhao, H.; Cai, H. Power decoupling strategy based on 'virtual negative resistor' for inverters in low-voltage microgrids. IET Power Electron. 2016, 9, 1037-1044. [CrossRef]

15. Wu, X.; Shen, C.; Iravani, R. Feasible Range and Optimal Value of the Virtual Impedance for Droop-Based Control of Microgrids. IEEE Trans. Smart Grid 2016, 8, 1242-1251. [CrossRef]

16. Zhu, Y.; Zhuo, F.; Wang, F.; Liu, B. A Wireless Load Sharing Strategy for Islanded Microgrid Based on Feeder Current Sensing. IEEE Trans. Power Electron. 2015, 30, 6706-6719. [CrossRef]

17. Mahmood, H.; Michaelson, D.; Jiang, J. Accurate Reactive Power Sharing in an Islanded Microgrid Using Adaptive Virtual Impedances. IEEE Trans. Power Electron. 2015, 30, 1605-1617. [CrossRef]

18. He, J.; Li, Y.W.; Blaabjerg, F. An Enhanced Islanding Microgrid Reactive Power, Imbalance Power, and Harmonic Power Sharing Scheme. IEEE Trans. Power Electron. 2015, 30, 3389-3401. [CrossRef]

19. Vandoorn, T.L.; Meersman, B.; Kooning, J. Analogy between Conventional Grid Control and Islanded Microgrid Control Based on a Global DC-Link Voltage Droop. IEEE Trans. Power Deliv. 2012, 27, 1405-1414. [CrossRef]

20. Guerrero, J.M.; Matas, J.; de Vicuña, L.G. Decentralized Control for Parallel Operation of Distributed Generation Inverters in Microgrids Using Resistive Output Impedance. IEEE Trans. Ind. Electron. 2007, 54, 994-1004. [CrossRef]

21. Yan, X.; Zhang, Y. Power Coupling Analysis of Inverters based on Relative Gain Method and Decoupling Control based on Feedforward Compensation. In Proceedings of the Renewable Power Generation, Beijing, China, 17-18 October 2015. 